\section{PREVALENCE OF EXPOSURE TO OCCUPATIONAL CARCINOGENS AMONG ETHNIC MINORITY WORKERS IN AUSTRALIA}

${ }^{1}$ Terry Boyle, ${ }^{1}$ Renee Carey, ${ }^{2}$ Deborah Glass, ${ }^{1}$ Susan Peters, ${ }^{1}$ Lin Fritschi, ${ }^{1}$ Alison Reid. ${ }^{1}$ The University of Western Australia, Perth, Western Australia, Australia; ${ }^{2}$ Monash University, Melbourne, Victoria, Australia

\subsection{6/oemed-2014-102362.49}

Objectives Although several studies have estimated the prevalence of occupational carcinogens in the general population, little is known about exposure to occupational carcinogens among ethnic minority workers. The aims of this study were to: estimate the prevalence of occupational exposure to carcinogens among ethnic minority workers in Australia; and compare their exposure prevalence to that of the general Australian-born working population ('Australian workers').

Method This was a cross-sectional telephone-based survey conducted in Australia in 2013. Participants were aged 18 to 65 years, of Arabic, Chinese or Vietnamese ancestry, and currently employed. Assessment of occupational exposures classified participants as unexposed, possibly exposed or probably exposed to each of 38 occupational carcinogens. Modified Poisson regression determined whether the workers in this study were more likely to be exposed to carcinogens than Australian workers.

Results Of the 749 participants, 31.6\% were assessed as being probably exposed to at least one carcinogen. Controlling for confounders, ethnic minority workers were less likely to be exposed to occupational carcinogens than Australian workers $(\mathrm{RR}=0.88,95 \% \mathrm{CI}=0.80-0.96)$. For specific carcinogens, compared with Australian workers, overseas-born Chinese workers were significantly more likely to be exposed to PAHs, Australian-born Arab workers were significantly more likely to be exposed to environmental tobacco smoke, and all Arab workers were significantly more likely to be exposed to silica.

Conclusions Approximately one-third of the ethnic minority workers in this study were exposed to carcinogens. They were less likely to be exposed than Australian workers overall; however for specific carcinogens exposure was more likely, depending on country of birth.

\section{PREVALENCE OF EXPOSURE TO SOME OCCUPATIONAL CARCINOGENS IN FRANCE: EVOLUTION BETWEEN 1999 AND 2007}

'Marie Houot, 1,2Corinne Pilorget, 1,2Brigitte Dananché, 'Laurène Delabre, 1,35téphane Ducamp, 'Loïc Garras, 'Danièle Luce, 'Mounia El Yamani. 'French Institute for Public Health Surveillance, Occupational Health Department, Saint-Maurice, France; ${ }^{2}$ Claude Bernard Lyon University, Epidemiological Research and Surveillance Unit in Transport, Occupation and Environment, Lyon, France; ${ }^{3}$ Bordeaux Segalen University, Associated Team in Occupational Health, Bordeaux, France

\subsection{6/oemed-2014-102362.50}

Objectives To use job-exposure matrices (JEM), as a tool to describe trends of occupational exposure to carcinogenic chemicals present in the French workplace.

Method MATGÉNÉ JEMs assess for each job and a given period in France, several exposure indices such as probability, intensity and frequency of exposure. Linking these matrices with job information data coming from French population allow to estimate the prevalence of workers exposed to several chemicals for a given year. To study the evolution of exposure to carcinogens, prevalence of exposure in France for the years 1999 and 2007 were estimated from the population census of 1999 and from a representative sample of the population in 2007.

Results French available JEMs assess the exposure of workers since the 1950s for various occupational carcinogens: crystalline silica, benzene, trichlorethylene, perchlorethylene, leather dust, asbestos and refractory ceramic fibres (RCF). A significant decrease in the prevalence of exposure between 1999 and 2007 was observed among men for silica with 7.4\% and 5.6\% respectively, asbestos with $6.3 \%$ and $1.1 \%$ and RCF with $0.5 \%$ and $0.3 \%$. For women, a significant decrease was also noticed for exposure to asbestos and leather dust. For solvents, the prevalence of exposure remained stable in both men and women.

Conclusions The proportion of workers exposed to carcinogens, particularly to asbestos, has decreased in France since 1999. However a substantial number of workers are still exposed. As complete occupational histories are available in the 2007 population sample, the JEMs will also be used to estimate lifetime exposure prevalence and the associated disease burden.

\section{ACUTE COGNITIVE EFFECTS OF MRI RELATED MAGNETIC FIELDS: THE ROLE OF VESTIBULAR SENSITIVITY}

${ }^{1}$ Lotte van Nierop, ${ }^{1}$ Pauline Slottje, ${ }^{1}$ Matine van Zandvoort, ${ }^{2}$ Herman Kingma, ${ }^{1}$ Hans Kromhout. ${ }^{1}$ Utrecht University, Utrecht, The Netherlands; ${ }^{2}$ University Hospital Maastricht, Maastricht, The Netherlands

\subsection{6/oemed-2014-102362.51}

Objectives Movement in the magnetic fields around MRI systems showed acute negative effects on concentration, memory, visuo-spatial orientation and postural body sway. A crucial role of the vestibular system has been hypothesised. We aimed to gain more insight whether subjects with a relatively (un)sensitive vestibular system performed differently on cognitive tasks when (moving) in a the static magnetic field of an MRI scanner.

Method In a double blind randomised cross over experiment 36 healthy volunteers underwent several cognitive tasks in 4 experimental sessions. Two were exposure conditions near a 7 Tesla (T) MRI system with personal exposure of $1.0 \mathrm{~T}$. In one of these conditions additional time-varying magnetic fields of $2.4 \mathrm{~T} / \mathrm{s}$ were induced by making standardised head movements. Of the two sham conditions $(0 \mathrm{~T})$ one was with and the other without such head movements. Vestibular sensitivity of each subject was assessed by the rotary chair test, the caloric reflex test and selfreported sensitivity to motion sickness.

Results Linear mixed models are currently in progress to test cognitive performance in a magnetic field for subjects with a low, normal and high sensitive vestibular organ. Preliminary results seem to suggest some differential cognitive effects of magnetic field exposure according to relative vestibular sensitivity. Further results will be presented at the conference.

Conclusions These findings are important to better understand a possible working mechanism evoking these cognitive effects. Moreover, these finding can form a basis for the design of relevant protective and precautionary control measures for employees working close to an MRI system. 